\title{
LINEAR ARRAY Human Papillomavirus Genotyping Test Amenable to Automation by Implementation of GT-Blot 48 - A Pilot Study
}

\author{
Michael Schmid*, Gunnar Schalasta, Andrea Speicher and Gisela Enders
}

\author{
Institute for Virology, Infectiology and Epidemiology e.V., Rosenbergstraße 85, D-70193 Stuttgart, Germany
}

\begin{abstract}
Objectives: Persistent infection with high-risk human papillomavirus is associated with the development of cervical intraepithelial neoplasia and a key factor in progression to invasive cancer. The Linear Array HPV genotyping test (LA) is a valuable diagnostic tool enabling type-specific detection of DNA from 37 anogenital high- and low-risk HPV genotypes. Its value, however, is hampered by the underlying labour-intensive manual line blot technology. This study was designed to improve the suitability of LA in routine use by implementing the GT-Blot 48 instrument as an alternative instead of using the proposed manual detection protocol.

Methods: Automation of LA processing was evaluated by comparing GT-blot 48 processed strips to the manufacturer recommended manual protocol. For type-specificity we compared the performance of GT-blot 48 processed strips with that of PapilloCheck, another PCR-based HPV typing assay. Furthermore, the performance of HPV DNA detection by GTblot 48 processed strips to that by Hybrid Capture 2 ( $\mathrm{hr}-\mathrm{HC} 2$ ) assay was compared, using samples from 122 women attending routine cervical examination.

Results: GT-blot 48 processed strips and the manual method demonstrate equal performance. The overall agreement of HPV prevalence between automated LA and PapilloCheck was 96.6\% (kappa = 0.92). A 91.2\% concordance was observed between automated LA and hr-HC2 (kappa $=0.82)$.

Conclusions: Automated LA processing is a valuable alternative to the manual method. Results are highly comparable with those obtained by hr-HC2 and PapilloCheck. Implementation of GT-blot 48 is a reliable easy-to-use format, which offers increased time efficiency and the additional benefit of high-throughput with standardized processing.
\end{abstract}

Keywords: HPV persistence, cervical cancer.

\section{INTRODUCTION}

Although the majority of HPV infections are benign, most often transient and self-limited, it is widely accepted that persistent type-specific HPV infection is a necessary event in cervical cancer precursor lesion and invasion [1-5]. More than 100 different HPV genotypes have been identified and classified as high- or low-risk, based on their association with cervical lesions $[6,7]$. The most common clinically important types are the high-risk HPV genotypes (i.e., types $16,18,31,33,35,39,45,51,52,56,58,59,68,73$, and 82) which can be found in virtually all cervical cancer [8-10].

Sensitive and reliable HPV tests have been wellestablished in cervical cancer screening like HC2 (Digene Corporation, Gaithersburg, MD) [11-13] and AMPLICOR HPV test (Roche Diagnostics, Mannheim, Germany) [14-16] with the limitation that none of these assays provide information on the specific HPV type present in the patient sample. However, type-specific results provide additional information for clinicians regarding both follow up treatment [17, $18]$ and vaccination.

At present, two widely used HPV genotyping assays are on the market, among them the $\mathrm{SPF}_{10}$-LiPA HPV genotyping

*Address correspondence to this author at the Institute for Virology, Infectiology and Epidemiology e.V., Rosenbergstraße 85, D-70193 Stuttgart, Germany; E-mail: schmid@labor-enders.de test [19-22] and the Roche HPV LINEAR ARRAY (Roche Diagnostics, Mannheim, Germany) [23, 24]. The LA assay amplifies a fragment from the L1 region of HPV by using a broad-spectrum PCR primer set [25]. Despite its widespread use, however, its value is compromised both by the cumbersome DNA extraction protocol and the labour-intensive manual reverse line blot technology.

For LA the MagNA Pure LC protocol was recently adapted for automated HPV DNA isolation instead of the manual AmpliLute kit (Qiagen, Inc., Valencia, CA) [24-27]. On the basis of these promising results, we sought to automate the back-end detection of LA by implementation of the GT-blot 48 instrument (Bee Robotics Ltd., Cibyn Industrial Estate, Caernarfon, N. Wales, UK). This bench-top instrument is designed for use with line blot strip assay eliminating the need for tedious washing and hybridization steps.

The present study was designed (i) to automate the backend detection of LA, (ii) to analyze its robustness in the setting of a routine diagnostic laboratory, and (iii) to compare the detection of HPV DNA by GT-blot 48 processed strips to that by hr-HC2 and PapilloCheck (Greiner Bio-One, Frickenhausen, Germany).

\section{MATERIALS AND METHODS}

\section{Clinical Specimens}

Epithelial specimens from 122 women attending routine cervical examination were collected by DNA Pap HC cervi- 
cal sampler (Digene), suspended in $1 \mathrm{ml}$ of ViraPap/Viratype transport medium (Digene) and submitted to the laboratory for HPV detection by hr-HC2. The median age was 36 years, and the range 17 to 63 years. According to previous cytologic results, $34 \%(n=41)$ showed cytological abnormalities; $23 \%(\mathrm{n}=28)$ with low-grade squamous intraepithelial lesions (LSILs), 6\% $(\mathrm{n}=7)$ with atypical squamous cells (ASCs), and $5 \%(n=6)$ with high-grade squamous intraepithelial lesions (HSILs).

\section{HC2}

The FDA-approved HC2 is a signal amplification assay that uses antibody capture of HPV DNA and RNA probe hybrids and chemiluminescence signal detection. The HC2 includes a mixture of probes for the following high-risk (hr) cancer-associated HPV genotypes (types 16, 18, 31, 33, 35, $39,45,51,52,56,58,59$, and 68). The low-risk probe set for HPV genotypes (types 6, 11, 42, 43, 44) was not used. All samples with a relative light units/cutoff (RLU/CO) ratio of $>1.00$ were considered positive. The HC2 was performed according to the manufacturer's instructions.

\section{MagNA Pure}

Automated sample preparation was done on the MagNA pure (MP) instrument by using the MagNA Pure LC Total Nucleic Acid Isolation kit (Roche Applied Science, Mannheim, Germany), as described by the manufacturer. In brief, total DNA was isolated from $200 \mu 1$ of a ViraPap/Viratype aliquot by using the MP. A negative control was included in each DNA extraction run to monitor the DNA isolation procedure. DNA was resuspended in $120 \mu \mathrm{l}$ of PCR-grade $\mathrm{H}_{2} \mathrm{O}$, and stored at $-20^{\circ} \mathrm{C}$ until further processing by LA and PapilloCheck.

\section{LINEAR ARRAY}

The LA HPV genotyping test is based on reverse hybridization of amplicons to immobilized membrane-bound probe. HPV DNA was detected in cervical samples by multiplex PCR targeted to the conserved L1 region of the viral genome. Also, the system includes co-amplification of the human $\beta$-globin gene as an internal control. PCR was performed in a final volume of $100 \mu \mathrm{l}$ with $50 \mu \mathrm{l}$ of kit supplied master mix and $50 \mu \mathrm{l}$ of extracted DNA, following the manufacturer's instructions. After amplification, the biotinylated PCR products were denatured immediately by addition of $100 \mu \mathrm{l} \mathrm{NaOH}$. Stringent hybridization of the denatured amplicons $(75 \mu \mathrm{l})$ and genotyping using the LA protocol were performed on the GT-Blot 48 as described below. Only samples positive for $\beta$-globin were considered for analysis. LA genotyping strips were interpreted using the HPV reference guide provided by the manufacturer.

\section{GT-Blot 48}

The GT-blot 48 is designed for use of DNA STRIP technology-based reverse hybridization kits including the screening of human polymorphisms (e.g. Factor V Leiden or hereditary haemochromatosis) and identification of microbiological organisms (e.g. methicillin-resistant Staphylococcus aureus). The instrument is equipped with a moving arm to both aspirate and dispense reagents across the tray, 7 peristaltic pumps on board, 2 built in pre-heater for warming up reagents prior to being dispensed into the tray, and also with a waste tube. The detection is performed within a 48 well tray, which is held on a heatable platform to ensure uniform temperature control during the different steps of the assay. The minimum number of samples that can be processed during a run is 2 and the maximum is 48 at a time. The GT-blot 48 can be programmed from a PC using the supplied editor software for individual application requirements and downloaded onboard for future use. Stored programs can be selected for use via the GT-blot 48 integral keypad. The temperature setting is ambient to $55^{\circ} \mathrm{C}$. At the end of the assay the instrument is cleaned with two pre-programmed settings. In addition, the trays should be cleaned with a washing solution (e.g. incubation of 5\% SDS for at least 2 hours and subsequently purification of the wells with a brush, followed by a rinse with de-ionised water). The tubing of the instrument should be flushed with $0.5 \%$ chloric solution, followed by de-ionised water.

\section{PapilloCheck DNA Chip}

The PapilloCheck test kit is intended to be used for the detection and differentiation of 24 types of HPV in clinical specimens (Greiner Bio-One). The assay was performed according to the manufacturer's instructions. The assay principle is based on the detection of the E1 gene from HPV, amplified and fluorescence-labelled (Cy5) in the presence of specific fluorophore-labelled primers. In the same reaction a fragment of the human ADAT1 gene is amplified to monitor DNA extraction and template quality for the PCR. The amplified products are then hybridized to complementary DNAprobes present in replicates of 5 on each array. For one reaction the following components were mixed in a total volume of $25 \mu \mathrm{l}$ : $19.8 \mu \mathrm{l}$ PapilloCheck mastermix, $0.2 \mu \mathrm{l}$ AmpliTaq Gold polymerase (5U/ $\mu 1$; Applied Biosystems, Branchburg, NJ), $5 \mu \mathrm{l}$ extracted DNA. The amplification steps were 10 min of initial denaturation at $95^{\circ} \mathrm{C}$, followed by 40 cycles with $25 \mathrm{~s}$ of annealing at $55^{\circ} \mathrm{C}, 45 \mathrm{~s}$ of elongation at $72^{\circ} \mathrm{C}$, and $30 \mathrm{~s}$ of denaturation at $95^{\circ} \mathrm{C}$. An additional 2-step cycling followed by 15 cycles with $45 \mathrm{~s}$ of annealing/extension at $72^{\circ} \mathrm{C}$, and $30 \mathrm{~s}$ of denaturation at $95^{\circ} \mathrm{C}$. After amplification, $5 \mu 1$ of the PCR-products were mixed with $30 \mu 1$ PapilloCheck Hybridization buffer, and $25 \mu \mathrm{l}$ of the mix transferred into each compartment of the chip. After hybridization at room temperature in a humid atmosphere, the chips were subsequently washed at room temperature in wash solution I for $10 \mathrm{~s}$, at $50^{\circ} \mathrm{C}$ in wash solution II for $60 \mathrm{~s}$, followed by the final washing step at room temperature for $10 \mathrm{~s}$. The PapilloCheck DNA-chips are scanned with the CheckScanner at excitation wavelengths of 532 and $635 \mathrm{~nm}$ as directed by the manufacturer. To control the hybridization reaction, the Cy3labelled probe in the hybridization buffer will react with the adequate complementary DNA probes spotted on the chip. Analysis and acceptance of individual results were done automatically with the CheckReport software (version 2.0.3).

\section{Reference Samples}

A panel of samples from an external quality assurance program, including HPV16, -18 , and -6 and a HPV negative control probe (MRC-5 cells) were used as reference (INSTAND, Düsseldorf, Germany). 


\section{Assessment of Results}

For direct comparison between hr-HC2 and LA only the assay common high-risk HPV genotypes (types 16, 18, 31, $33,35,39,45,51,52,56,58,59$ and 68 ) have been considered for assessment of the results. Likewise, to compare between LA and PapilloCheck, only the assay common genotypes (types $6,11,16,18,31,33,35,39,40,42,45,51$, $52,53,55,56,58,59,66,68,70,73$ and 82) are considered. Assay unique genotypes are presented in Table $\mathbf{1 .}$

Table 1. Overview of Assay Unique HPV Genotypes

\begin{tabular}{|c|c|c|c|}
\hline HPV Type & $\mathrm{HC} 2$ & $\mathbf{L A}$ & PapilloCheck \\
\hline 6 & $\mathrm{X}$ & $\mathrm{X}$ & $\mathrm{X}$ \\
\hline 11 & $\mathrm{X}$ & $\mathrm{X}$ & $\mathrm{X}$ \\
\hline 16 & $\mathrm{X}$ & $\mathrm{X}$ & $\mathrm{X}$ \\
\hline 18 & $\mathrm{X}$ & $\mathrm{X}$ & $\mathrm{X}$ \\
\hline 26 & & $\mathrm{X}$ & \\
\hline 31 & $X$ & $\mathrm{X}$ & $X$ \\
\hline 33 & $\mathrm{X}$ & $\mathrm{X}$ & $\mathrm{X}$ \\
\hline 35 & $\mathrm{X}$ & $\mathrm{X}$ & $\mathrm{X}$ \\
\hline 39 & $\mathrm{X}$ & $\mathrm{X}$ & $X$ \\
\hline 40 & & $\mathrm{X}$ & $\mathrm{X}$ \\
\hline 42 & $\mathrm{X}$ & $\mathrm{X}$ & $\mathrm{X}$ \\
\hline 43 & $\mathrm{X}$ & & $\mathrm{X}$ \\
\hline 44 & $\mathrm{X}$ & & $\mathrm{X}$ \\
\hline 45 & $\mathrm{X}$ & $\mathrm{X}$ & $\mathrm{X}$ \\
\hline 51 & $X$ & $\mathrm{X}$ & $\mathrm{X}$ \\
\hline 52 & $X$ & $\mathrm{X}$ & $X$ \\
\hline 53 & & $\mathrm{X}$ & $X$ \\
\hline 54 & & $\mathrm{X}$ & \\
\hline 55 & & $\mathrm{X}$ & $\mathrm{X}$ \\
\hline 56 & $\mathrm{X}$ & $\mathrm{X}$ & $\mathrm{X}$ \\
\hline 58 & $\mathrm{X}$ & $\mathrm{X}$ & $\mathrm{X}$ \\
\hline 59 & $\mathrm{X}$ & $\mathrm{X}$ & $\mathrm{X}$ \\
\hline 61 & & $\mathrm{X}$ & \\
\hline 62 & & $\mathrm{X}$ & \\
\hline 64 & & $\mathrm{X}$ & \\
\hline 66 & & $\mathrm{X}$ & $X$ \\
\hline 67 & & $\mathrm{X}$ & \\
\hline 68 & $\mathrm{X}$ & $\mathrm{X}$ & $\mathrm{X}$ \\
\hline 69 & & $\mathrm{X}$ & \\
\hline 70 & & $\mathrm{X}$ & $\mathrm{X}$ \\
\hline 71 & & $\mathrm{X}$ & \\
\hline 72 & & $\mathrm{X}$ & \\
\hline 73 & & $\mathrm{X}$ & $\mathrm{X}$ \\
\hline 81 & & $\mathrm{X}$ & \\
\hline 82 & & $\mathrm{X}$ & $\mathrm{X}$ \\
\hline 83 & & $X$ & \\
\hline 84 & & $\mathrm{X}$ & \\
\hline IS39 & & $\mathrm{X}$ & \\
\hline CP6108 & & $\mathrm{X}$ & \\
\hline
\end{tabular}

The genotyping results were termed as follows: concordant (both assays showed identical assay common genotypes), compatible (both assays showed at least one or more assay common genotype(s)), and discordant (no assay common genotype similarities).

\section{Statistical Analysis}

We compared the agreement between the used methods by calculating kappa statistics and $95 \%$ confidence intervals (CIs).

\section{RESULTS}

Precision of automated LA processing was determined by performing replicate testing. Within-run and between-run variations were determined using a panel of reference probes containing one HPV-negative sample (MRC-5-cells) and three HPV-positive samples (HPV6, -16, and -18). Withinrun and between-run variations were performed in duplicate. The negative control was negative and the positive probes gave the expected band pattern.

Comparison of manual to automated method was done by retesting 20 cervical specimens in a blinded manner. The testing comprised $10 \mathrm{HPV}$-negative and $10 \mathrm{HPV}$-positive samples containing 8 single infections with HPV types 16 , $18,31,39,42,53,56$ and 84 and 2 multiple infections with HPV6 plus HPV16 and HPV31 plus HPV35 plus HPV73. We found completely identical results by comparing the automated GT-blot 48 processed strips to the manufacturer recommended manual method.

Next, we compared the type-specific performance of GTblot 48 processed strips with the PapilloCheck DNA chip, a different HPV genotyping method (Table 2). To increase the number of HPV types evaluated, PapilloCheck was applied to 18 specimens that tested positive for HPV by hr-HC2 and LA (single and multiple-type) and to 12 randomly selected hr-HC2-negative samples. One sample was excluded from the analysis due to both a negative internal amplification control and negative HPV result by PapilloCheck. The overall agreement of HPV prevalence for the remaining 29 valid samples between the two methods was $96.6 \%$ (28/29) of the samples $(\mathrm{kappa}=0.92)$. Of the 18 LA-positive samples with assay common genotypes, 12/18 (67\%) were concordant, $5 / 18(28 \%)$ compatible, and only $1 / 18(5 \%)$ showed discordant results (Table 3 ). The proportion of types detected by LA and PapilloCheck differ, 25 genotypes compared to 22 genotypes, respectively. Importantly, all 5 compatible results were multiple-type infections. In one case two HPV low-risk types were missed by PapilloCheck (HPV53 plus HPV73), and in two cases one high-risk type (HPV18 and HPV51). Conversely, LA failed to detect high-risk type HPV68 in two cases. One sample gave a discrepant result between the two assays. The PapilloCheck did not detect a single-type infection of HPV high-risk (HPV58), which was found by LA. Unfortunately, due to a limitation in sample availability, this result could not be resolved by retesting the sample.

Finally, we evaluated the concordance of HC2 and automated LA in detecting high-risk HPV among 122 unselected specimens representing various degrees of dysplasia (Table 4). All specimens tested positive for human B-globin internal control and were considered valid. Of the 122 specimens, 9 
Table 2. Analysis of Concordance Between LA and PapilloCheck $^{\mathrm{a}}$

\begin{tabular}{|c|c|c|c|}
\hline \multirow{2}{*}{ LA Result } & \multicolumn{3}{|c|}{ No. of Samples Tested by PapilloCheck } \\
\cline { 2 - 4 } & Negative & Positive & Total \\
\hline \hline Negative & 11 & 0 & 11 \\
\hline Positive & 1 & 17 & 18 \\
\hline Total & 12 & 17 & 29 \\
\hline
\end{tabular}

70,73 , and 82 (common genotypes).

Table 3. HPV Type-Specific Results with LA and PapilloCheck

\begin{tabular}{|c|c|c|c|}
\hline \multirow{2}{*}{ Case No. } & \multicolumn{2}{|c|}{ HPV Genotype } & \multirow{2}{*}{ Classification } \\
\cline { 2 - 4 } & Linear Array & PapilloCheck & \\
\hline \hline 1 & 16,68 & 16,68 & Concordant \\
\hline $\mathbf{2}$ & 18,51 & 18 & Compatible \\
\hline 3 & 16 & 16 & Concordant \\
\hline 4 & 16 & 16 & Concordant \\
\hline 5 & 56 & 56 & Concordant \\
\hline 6 & 58 & - & Discordant \\
\hline 7 & 31 & 31 & Concordant \\
\hline 8 & 39 & 39 & Concordant \\
\hline 9 & 56 & 56 & Concordant \\
\hline $\mathbf{1 0}$ & 16 & 16,68 & Compatible \\
\hline $\mathbf{1 1}$ & 16,18 & 16 & Compatible \\
\hline 12 & 16 & 16 & Concordant \\
\hline $\mathbf{1 3}$ & 31 & 31,68 & Compatible \\
\hline $\mathbf{1 4}$ & $40,52,53,56,73$ & $40,52,56$ & Compatible \\
\hline 15 & 70 & 70 & Concordant \\
\hline 16 & 35 & 35 & Concordant \\
\hline 17 & 70 & 70 & Concordant \\
\hline 18 & 16 & 16 & Concordant \\
\hline
\end{tabular}

Note: Boldface denotes compatible results with assay common genotypes.

were excluded since hr-HC2 was negative and LA detected an assay unique genotype $(\mathrm{n}=113)$. The agreement between the two assays for assay common genotypes was $91.2 \%$, and the kappa value was 0.82 (95\% CI, 0.72 to 0.93$)$. Among the 59 hr-HC2 positive samples, 51 were positive by LA, whereas two samples were LA positive but hr-HC2 negative. Seven of eight hr-HC2-only-positive samples were positive for the assay unique low-risk genotypes HPV53, HPV70 and IS39. The most frequent high-risk HPV types detected were HPV16 $(\mathrm{n}=15) 12 \%$, HPV51 $(\mathrm{n}=9) 7 \%$, and HPV31 $(\mathrm{n}=8) 6 \%$.

\section{DISCUSSION AND CONCLUSIONS}

Laboratory automation has simplified clinical diagnostics while heightening their degree of standardization, efficiency, and capacity. Our goal was to automate the back-end detection of LA by implementation of GT-blot 48. We have successfully automated LA without any modifications like dispense volumes, incubation time and wash parameters. The hands on time was reduced from 120 to 30 minutes between the manual and automated detection. Based on the testing of 8 patients, the total time to complete LA was $7 \mathrm{~h}$, thereby allowing laboratory to provide the result to the clinician in a timely fashion.

Table 4. Analysis of Concordance Between hr-HC2 and $\mathrm{LA}^{\mathrm{a}}$

\begin{tabular}{|c|c|c|c|}
\hline \multirow{2}{*}{ LA Result } & \multicolumn{3}{|c|}{ No. of Samples Tested by hr-HC2 } \\
\cline { 2 - 4 } & Negative & Positive & Total \\
\hline \hline Negative & 52 & 8 & 60 \\
\hline Positive & 2 & 51 & 53 \\
\hline Total & 54 & 59 & 113 \\
\hline
\end{tabular}

We found that GT-blot 48 processed strips were specific and showed $100 \%$ concordance with results from the recommended manual protocol. Type-specific performance was evaluated by comparing results of GT-blot 48 processed strips with results from PapilloCheck. Currently, there is no gold standard test available for identification of HPV. To increase the discriminatory power, we choose the PapilloCheck instead of the widely used $\mathrm{SPF}_{10}$-HPV-LiPA system as a reference assay, since the PCR primer of the $\mathrm{SPF}_{10}$ testing system and LA bind within the conserved L1 open reading frame. The overall agreement of HPV prevalence between LA and PapilloCheck was 96.6\%. Similarly, we found an almost perfect agreement, when type-specific results were classified according to cancer-associated HPV types, with at least one positive carcinogenic HPV type. Both HPV PCRbased typing systems showed different profiles for the detection of HPV genotypes within compatible results. The difference in analytical sensitivity may be due to competition for PCR primers in the presence of multiple HPV types, which is consistent with the findings of other studies [20, 22]. Indeed, all of the compatible results are multiple-type infections. One sample was LA positive but negative by hr-HC2 and PapilloCheck. Interpretation of this result is complicated by several factors. As indicated by the manufacturer, the limit of detection of HPV58 is comparable. On the other hand, contamination is a less likely explanation, given that the negative controls included in each run were found consistently negative and no other HPV58 isolate (a possible source of contamination) was detected during our study. Together with cytological findings, this sample was assumed to be truly positive.

The strength of agreement between hr-HC2 and LA is considered almost perfect (91.2.\% agreement; kappa $=0.82)$. A recent study reported a similar agreement of results $(91 \%$; kappa $=0.81)$ [27]. Possible explanations for the discordant results by hr-HC2 and LA could be related to: (1) stochastic sampling error (i.e. where samples with low HPV DNA concentration will not always allow positive aliquots to be made), and (2) cross-reactivity of HC2 with other HPV types not included in the hr-HC2 assay probe set. It is important to note that 6 of $8 \mathrm{hr}-\mathrm{HC} 2$ positive samples were positive for the assay unique low-risk type HPV70 and HPV53. The HC2 cross-reactivity with noncarcinogenic HPV observed here is consistent with previous reports $[18,28]$.

There are some limitations inherent to our study. First, the analysis consisted of women from the general population 
representing a low HPV prevalence. The impact, if any, of a high prevalence population on type-specificity should be further studied. Nevertheless, the obtained HPV genotypes provided some insight into the excellent concordance between the two genotyping methods. Second, cytological data available to us are incomplete and precluded a detailed analysis. A recent study, however, using the LA assay, demonstrated a strong association of increasing severity of cytologic interpretation with more-carcinogenic HPV risk categories [23].

Back-end automation of LA is an effective alternative to the manual detection protocol. Our results indicate the validity of the automated platform. The chief features of the assay are its reduced hands-on technician time and its potential for high-throughput. At present, there is a need for type-specific information to manage viral persistence before precancerous lesions develop into progressive disease [29, 30] and to monitor the risk of replacement with non-vaccine types [31].

\section{ACKNOWLEDGEMENTS}

We thank for expert technical assistance with the GTBlot 48 Annette Arents and Ralph Hahn.

\section{REFERENCES}

[1] Muñoz N, Bosch FX, de Sanjose S, et al. The causal link between human papillomavirus and invasive cervical cancer: a populationbased cause-control study in Columbia and Spain. Int J Cancer 1992; 52: 743-9.

[2] Cuzick J, Terry G, Ho L, et al. Type-specific human papillomavirus DNA in abnormal smears as a predictor of high-grade cervical intraepithelial neoplasia. Br J Cancer 1994; 69: 167-71.

[3] Ho GY, Burk RD, Klein S, et al. Persistent genital human papillomavirus infection as a risk factor for persistent cervical dysplasia. J Natl Cancer Inst 1995; 87: 1365-71.

[4] Remmink AJ, Walboomers JM, Helmerhorst TJ, et al. The presence of persistent high-risk HPV gentoypes in dysplastic cervical lesions is associated with progressive disease: natural history up to 36 month. Int J Cancer 1995; 61: 306-11.

[5] Bosch FX, Lorincz A, Muñoz N, Meijer CJ, Shah KV. The causal relation between human papillomavirus and cervical cancer. J Clin Pathol 2002; 55: 244-65.

[6] Muñoz N, Bosch FX., de Sanjose S, et al. Epidemiologic classification of human papillomavirus types associated with cervical cancer. N Engl J Med 2003; 348: 518-27.

[7] de Villiers EM, Fauquet C, Broker TR, Bernard HU, Zur Hausen H. Classification of papillomaviruses. Virology 2004; 324: 17-27.

[8] Bosch FX, Manos MM, Muñoz N, et al. Prevalence of human papillomavirus in cervical cancer: a worldwide perspective. International biological study on cervical cancer (IBSCC) Study Group. J Natl Cancer Inst 1995; 87: 796-802.

[9] Walboomers JM, Jacobs MV, Manos MM, et al. Human papillomavirus is a necessary cause of invasive cervical cancer worldwide. $\mathbf{J}$ Pathol 1999; 189: 12-19.

[10] Clifford GM, Smith JS, Plummer M, Muñoz N, Franceschi S. Human papillomavirus types in invasive cervical cancer worldwide: a metaanalysis. Br J Cancer 2003; 88: 63-73.

[11] Poljak M, Brencic A, Seme K, Vince A, Marin IJ. Comparative evaluation of first and second-generation digene hybrid capture assays for detection of human papillomaviruses associated with high or intermediate risk for cervical cancer. J Clin Microbiol 1999; 37: 7967.

[12] Vince A, Kutela N, Iscic-Bes J, et al. Clinical utility of molecular detection of human papillomavirus in cervical samples by hybrid capture technology. J Clin Virol 2002; 25: S109-112.
[13] Castle PE, Wheeler CM, Solomon D, Schiffman M, Peyton CL. Interlaboratory reliability of Hybrid captures 2. Am J Clin Pathol 2004; 122: 238-45

[14] van Ham MA, Bakkers JM, Harbers GK, Quint WG, Massuger LF, Melchers WJ. Comparison of two commercial assays for detection of human papillomaviruses (HPV) in cervical scrape specimens: validation of the Roche AMPLICOR HPV test as a means to screen for HPV genotypes associated with a higher risk of cervical disorders. J Clin Microbiol 2005; 43: 2662-7.

[15] Sandri M, Lentati TP, Benini E, et al. Comparison of the Digene HC2 assay and the Roche AMPLICOR human papillomavirus (HPV) test for detection of high-risk HPV genotypes in cervical samples. J Clin Microbiol 2006; 44: 2141-6.

[16] Halfon P, Trepo E, Antoniotti G, et al. Prospective evaluation of the Hybrid Capture 2 and AMPLICOR human papillomavirus (HPV) tests for detection of 13 high-risk HPV genotypes in atypical squamous cells of uncertain significance. J Clin Microbiol 2007; 45: 313-6.

[17] Castle PE, Solomon D, Schiffman M, Wheeler CM. Human papillomavirus type 16 infections and 2-year absolute risk of cervical precancer in women with equivocal or mild cytologic abnormalities. J Natl Cancer Inst 2005; 97: 1066-71.

[18] Han J, Swan DC, Smith SJ, et al. Simultaneous amplification and identification of 25 human papillomavirus types with Templex technology. J Clin Microbiol 2006; 44: 4157-62.

[19] Melchers WJ, Bakkers JM, Wang J, et al. Short fragment polymerase chain reaction reverse hybridization line probe assay to detect and genotype a broad spectrum of human papillomavirus types. Clinical evaluation and follow-up. Am J Pathol 1999; 155: 1473-8.

[20] van Doorn L, Quint JW, Kleter B, et al. Genotyping of human papillomavirus in liquid cytology cervical specimens by the PGMY line blot assay and the SPF(10) line probe assay. J Clin Microbiol 2002; 40: 979-83

[21] Perrons C, Jelley R, Kleter B, Quint WG, Brink N. Detection of high risk human papillomavirus infections with hybrid capture II and SPF10/LiPA. J Clin Virol 2005; 32: 278-85.

[22] van Hamont D, van Ham MA, Bakkers JM, Massuger LF, Melchers W. Evaluation of the SPF10-INNO-LiPA human papillomavirus (HPV) genotyping test and the roche linear array HPV genotyping test. J Clin Microbiol 2006; 44: 3122-9.

[23] Castle PE, Sadorra M, Garcia F, Holladay EB, Kornegay J. Pilot study of a commercialized human papillomavirus (HPV) genotyping assay: comparison of HPV risk group to cytology and histology. J Clin Microbiol 2006; 44: 3915-7.

[24] Stevens MP, Rudland E, Garland SM, Tabrizi SN. Assessment of MagNA pure LC extraction for detection of human papillomavirus (HPV) DNA in PreservCyt samples by the Roche AMPLICOR and LINEAR ARRAY HPV test. J Clin Microbiol 2006; 44: 2428-33.

[25] Gravitt PE, Peyton CL, Alessi TQ, et al. Improved amplification of gential human papillomaviruses. J Clin Microbiol 2000; 38: 357-361.

[26] Schalasta G, Rosenthal T, Grothe M. Roche AMPLICOR human papillomavirus (HPV) and LINEAR ARRAY HPV test will profit from automated DNA extraction. Clin Lab 2007; 53: 131-3.

[27] de Antonio JC, Fernández-Olmos A, Mercadillo M, Lindemann ML, Mochales FB. Detection of high-risk human papillomavirus by two molecular techniques: hybrid capture and linear array. J Virol Methods 2008; 149: 163-6.

[28] Castle PE, Schiffman M, Burk RD, et al. Restricted cross-reactivity of hybrid capture 2 with nononcogenic human papillomavirus types. Cancer Epidemiol Biomarkers Prev 2002; 11: 1394-9.

[29] Wallin KL, Wiklund F, Angstrom T, et al. Type-specific persistence of human papillomavirus DNA before the development of invasive cervical cancer. N Engl J Med 1999; 341: 1633-8.

[30] Cuschieri KS, Whitley MJ, Cubie HA. Human papillomavirus type specific DNA and RNA persistence - implications for cervical disease progression and monitoring. J Med Virol 2004; 73: 65-70.

[31] Goldie SJ, Kohli M, Grima D, et al. Projected clinical benefits and cost-effectiveness of a human papillomavirus $16 / 18$ vaccine. J Natl Cancer Inst 2004; 96: 604-15. 